\title{
BMJ Open Population impact of different hypertension management guidelines based on the prospective population- based Heinz Nixdorf Recall study
}

\author{
Janine Gronewold (D) , ${ }^{1}$ Rene Kropp, ${ }^{1}$ Nils Lehmann, ${ }^{2}$ Andreas Stang, ${ }^{2,3}$ \\ Amir A Mahabadi, ${ }^{4}$ Christian Weimar, ${ }^{1}$ Martin Dichgans, ${ }^{5,6}$ Susanne Moebus, ${ }^{2}$ \\ Knut Kröger, ${ }^{7}$ Barbara Hoffmann, ${ }^{8}$ Karl-Heinz Jöckel, ${ }^{2}$ Raimund Erbel, ${ }^{2}$ \\ Dirk M Hermann, ${ }^{1}$ on behalf of the Heinz Nixdorf Recall Study Investigative Group
}

To cite: Gronewold J, Kropp R, Lehmann N, et al. Population impact of different hypertension management guidelines based on the prospective population-based Heinz Nixdorf Recall study. BMJ Open 2021;11:e039597. doi:10.1136/ bmjopen-2020-039597

- Prepublication history and additional material for this paper is available online. To view these files, please visit the journal online (http://dx.doi.org/10. 1136/bmjopen-2020-039597).

Received 20 April 2020 Revised 09 December 2020 Accepted 28 December 2020

Check for updates

(C) Author(s) (or their employer(s)) 2021. Re-use permitted under CC BY-NC. No commercial re-use. See rights and permissions. Published by BMJ.

For numbered affiliations see end of article.

Correspondence to

Dr Janine Gronewold;

janine.gronewold@uk-essen.de

\section{ABSTRACT}

Objective Hypertension guidelines strongly differ between societies. The current American College of Cardiology/American Heart Association (ACC/AHA) guideline recommends higher proportions of the general population for antihypertensive medication than the previous American and European guidelines. How cardiovascular risk differs between persons with and without antihypertensive medication recommendation has not been examined. Additionally, the population impact of American, European and international guidelines has not been compared systematically within the same study population.

Methods We compared the prevalence of antihypertensive medication recommendation according to the American (Joint National Committee on Prevention, Detection, Evaluation, and Treatment of High Blood Pressure 7 (JNC7), ACC/AHA 2017), European (European Society of Hypertension (ESH)/European Society of Cardiology (ESC) 2013/2018), and international (WHO/ International Society of Hypertension (ISH) 2003, ISH 2020) guidelines in 3092 participants of the population-based Heinz Nixdorf Recall study not taking antihypertensive medication at the baseline examination $(58.1 \pm 7.5$ years, $48.7 \%$ males). We furthermore compared incident cardiovascular events during the 5-year follow-up between participants with and without antihypertensive medication recommendation.

Results The ACC/AHA 2017 guideline recommended the highest percentage of participants for antihypertensive medication (45.8\%) compared with the JNC7 (37.2\%), ESH/ESC 2013 (17.8\%), ESC/ESH 2018 (26.7\%), WHO/ ISH 2003 (20.3\%) or ISH 2020 (25.0\%) guidelines. Participants with antihypertensive medication recommendation according to the ACC/AHA 2017 guideline had a significantly higher incidence of cardiovascular events during the 5-year follow-up compared with participants without this recommendation ( $2.5 \%$ vs $1.1 \%, p=0.003)$.

Conclusions Our results call for randomised controlled trials to investigate whether applying the stricter ACC/ AHA 2017 recommendation leads to a reduction in cardiovascular disease.

\section{Strengths and limitations of this study}

- We systematically compared the population impact (antihypertensive medication recommendation, blood pressure above treatment goal, incidence of cardiovascular events) of six different hypertension guidelines.

- Contrary to previous studies, guidelines from European, American and global societies were covered.

- Strengths of our study are the population-based sample, the state-of-the-art assessment of blood pressure and antihypertensive medication and the validation of cardiovascular events by an independent endpoint committee.

- A limitation of our study is its observational design, the lack of confounding control and thus lack of causal inference.

- Randomised controlled trials investigating whether earlier treatment of blood pressure leads to a reduction in cardiovascular disease are still lacking.

\section{INTRODUCTION}

The management of arterial hypertension plays a central role in the primary and secondary prevention of cardiovascular events. Guidelines for the diagnosis and treatment of arterial hypertension have been released by different societies. In 1977, The Joint National Committee on Prevention, Detection, Evaluation, and Treatment of High Blood Pressure (JNC) initiated hypertension guidelines, which progressively became the leading recommendation for hypertension management, even beyond the borders of the USA. ${ }^{1}$ To reflect current hypertension knowledge, the JNC guidelines have subsequently been updated every 4-6 years until the JNC7 guideline, which was published in $2003 .^{2}$ Following a longer gap of updates, the current American College of Cardiology/ 
American Heart Association (ACC/AHA) guideline was released in 2017. ${ }^{3}$ This ACC/AHA guideline acknowledged the increased risk of cardiovascular events in persons with mild or moderately elevated blood pressure $(\mathrm{BP})$, thus aiming at a reduction in cardiovascular burden in the general population. ${ }^{4}$ For Europe, the European Society of Hypertension (ESH)/European Society of Cardiology (ESC) guideline from 2013, ${ }^{5}$ which was updated in $2018,{ }^{6}$ provided treatment recommendations with higher BP thresholds than the ACC/AHA guideline. The ESH represents a sister society of the International Society of Hypertension (ISH), which released a global treatment guideline in 2003 together with the WHO. ${ }^{7}$ This global treatment guideline was updated in 2020 by the $\mathrm{ISH}^{8}$ with the novel aim to define optimal care as well as essential care in cases where optimal care is not faisible like in low-resource settings encountered in low-income and middle-income countries.

Since the guidelines from different societies strongly differ in their treatment recommendations and treatment goals, physicians are confronted with the problem of deciding which guideline to apply to which patient, ${ }^{9}$ leading to uncertainty, incapacity and inertia in the hypertension field. ${ }^{10}$ The population impact of different guidelines has so far not been systematically compared within the same population. In particular, the risk profile of persons recommended for treatment based on stricter BP goals, as suggested in the ACC/AHA 2017 guideline, ${ }^{3}$ remains unknown. In the prospective population-based Heinz Nixdorf Recall (HNR) study, we analysed (1) the frequencies and percentages of antihypertensive medication recommendations in participants hitherto not taking antihypertensive medication, (2) the frequencies and percentages of BP above treatment goal in participants taking antihypertensive medication based on different guidelines, and (3) the association of an antihypertensive medication recommendation with incident cardiovascular events during the 5-year follow-up.

\section{METHODS}

\section{Study cohort}

The HNR cohort is a random sample of 4814 men and women aged 45-75 years (online supplemental table S1) who were prospectively enrolled via mandatory citizen registries in Essen, Bochum and Mülheim/Ruhr, Germany, between December 2000 and August 2003 and received two follow-up examinations 5 and 10 years after the baseline examination. Mean $\pm \mathrm{SD}$ of 5 -year-follow-up examination time was $5.1 \pm 0.3$ years, follow-up response was $90.2 \%$. The study design has been described in detail elsewhere. ${ }^{11}$ All participants gave written informed consent.

\section{Assessment of BP and antihypertensive medication}

BP was measured with an automated oscillometric device (Omron 705-CP, Omron, Mannheim, Germany) and the mean value of the second and third of three measurements taken at least 2 min apart was used. Participants were asked to bring all the medications they had been taking during the 7 days prior to the examination appointment. Medications were coded according to the Anatomical Therapeutic Chemical Classification Index. ${ }^{12}$ Antihypertensive drug treatment was defined according to the Kooperative Gesundheitsforschung in der Region Augsburg (KORA) study definition. ${ }^{13}$ Measurement of further vascular risk factors needed to determine antihypertensive medication recommendation is described in the online supplemental methods.

\section{Assessment of incident cardiovascular events}

Cardiovascular events during follow-up comprised strokes (defined as focal neurological deficits over a period of $>24$ hours of presumed cerebrovascular origin), coronary events (including non-fatal acute myocardial infarction and coronary death defined as clinical symptoms, signs on electrocardiography, increased enzymes (levels of creatinine kinase), and troponin $\mathrm{T}$ or $\mathrm{I}$, as well, and necropsy changes), or independently coded causes of deaths according to chapter 9 of ICD-10. ${ }^{14}$

\section{Patient and public involvement}

Patients or the public were not involved in the design, conduct, reporting, or dissemination plans of our research.

\section{Hypertension guidelines}

We included suggestions about when to start antihypertensive treatment (antihypertensive medication recommendation) and which target $\mathrm{BP}$ to achieve with antihypertensive treatment (treatment goal) according to American (JNC7 and ACC/AHA 2017), European (ESH/ ESC 2013/2018) and international (WHO/ISH 2003 and ISH 2020) guidelines. An overview of antihypertensive medication recommendations for each of these guidelines in chronological order is shown in online supplemental figures S1-S6; treatment goals are specified in online supplemental table S2.

\section{Statistical analysis}

We analysed the frequencies and percentages of HNR participants hitherto not taking antihypertensive medication being recommended for antihypertensive medication according to the different hypertension management guidelines which were published after recruitment of the HNR study in 2003 (online supplemental figures S1-S6). In addition, we analysed the frequencies and percentages of HNR participants taking antihypertensive medication who still exhibited $\mathrm{BP}$ above the treatment goals suggested by the different hypertension management guidelines. We further compared baseline cardiovascular risk factors as well as incident cardiovascular events during the 5-year follow-up between participants with and without an antihypertensive medication recommendation. Continuous data are presented as mean \pm SD for normally distributed and median (IQR) for non-normally distributed data; categorical data are shown as frequencies 
(\%). Comparisons between participants with and without an antihypertensive medication recommendation were done with Student t-test for normally distributed data, Mann-Whitney U-test for non-normally distributed data, and $\chi^{2}$-test for categorical data. Cardiovascular event incidence rates for participants with and without an antihypertensive medication recommendation were adjusted for age and sex by standardisation to the sex (stratified into male and female) and age (stratified into 5-year intervals) distribution of the total cohort, 95\% CIs were calculated by the Clopper-Pearson method. Cases with missing values (number and percentages of missing values shown in online supplemental table S1 for each variable and in online supplemental table S3 for application of hypertension management guidelines) were excluded from analyses listwise. Except for common-carotid artery intima-media thickness (CIMT), which was used as a proxy marker of carotid atherosclerosis and is included in the WHO/ISH 2003 and ESH/ESC 2013 guidelines, missing values were rare, so that imputation of missing values was not required. The major reason for missing CIMT values was the limited availability of examiners or ultrasound equipment, especially in the initial months of this study $(\mathrm{n}=832)$. In a smaller proportion $(\mathrm{n}=347)$, CIMT could not be determined due to insufficient image quality. We also performed a sensitivity analysis excluding CIMT from the WHO/ISH 2003 and ESH/ESC 2013 guidelines.

For the implementation of the different hypertension management guidelines in the HNR cohort, we only included at least IIa class of recommendations with at least B level of evidence, for the ISH 2020 guideline we included the essential care. The term "or" consistently implies "and/or" for the description of hypertension management guidelines. Statistical analyses were performed using SPSS V.22 for Windows. ${ }^{15}$

\section{RESULTS}

\section{Study cohort}

The characteristics of the HNR cohort regarding variables that are included in the six American, European and international hypertension management guidelines published since recruitment of the HNR study cohort (online supplemental figures S1-S6) are shown in table 1, stratified by antihypertensive medication at the baseline examination. Since the baseline examination of the HNR cohort took place from December 2000 to August 2003, antihypertensive medication should have been recommended according to the JNC7, which represented the guideline then in force. The total HNR cohort comprises 4814 participants $(49.8 \%$ males, aged $45-75$ years, mean age 59.6 (SD 7.8) years). From this total cohort, 3099 participants were not taking antihypertensive medication at the baseline examination, 1699 participants were taking antihypertensive medication, and information was missing for 16 participants. For the analysis of antihypertensive medication recommendation, we included 3092 participants with valid BP data (table 1) of the 3099 HNR participants not taking antihypertensive medication at the baseline examination. For the analysis of BP above treatment goal, we included 1691 participants with valid BP data (table 1) of the 1699 HNR participants taking antihypertensive medication at the baseline examination. The characteristics of the total cohort and missing values not stratified according to antihypertensive medication at baseline are shown in online supplemental table S1.

Prevalence of antihypertensive medication recommendations and BP above treatment goal according to the different hypertension management guidelines

JNC7 2003 guideline

As already mentioned above, the JNC7 guideline was the predominating guideline in clinical settings at the time of the baseline examination of the HNR cohort in the USA and in Europe. Antihypertensive medication is recommended for all persons with systolic BP (SBP) $\geq 140 \mathrm{~mm}$ $\mathrm{Hg}$ or diastolic BP (DBP) $\geq 90 \mathrm{~mm} \mathrm{Hg}$ (online supplemental figure S1) as well as for persons at high cardiovascular disease risk (defined by the presence of diabetes mellitus or chronic kidney disease (CKD; see legend to online supplemental figure S1)) with $\mathrm{SBP} \geq 130 \mathrm{~mm} \mathrm{Hg}$ or $\mathrm{DBP} \geq 80 \mathrm{~mm} \mathrm{Hg}$ (online supplemental figure S1).

Among the 3092 participants from the HNR cohort who were not taking antihypertensive medication at the baseline examination, $37.2 \%$ were recommended for antihypertensive medication according to the JNC7 guideline (table 2). For antihypertensive medication, the JNC7 guideline suggests a treatment goal of SBP $<140 \mathrm{~mm}$ $\mathrm{Hg}$ and $\mathrm{DBP}<90 \mathrm{~mm} \mathrm{Hg}$ in all patients and a treatment goal of SBP $<130 \mathrm{~mm} \mathrm{Hg}$ and $\mathrm{DBP}<80 \mathrm{~mm} \mathrm{Hg}$ in patients suffering from diabetes mellitus or CKD (online supplemental table S2). Among the 1691 participants taking antihypertensive medication at the baseline examination, more than half $(56.4 \%)$ still exhibited BP values above the treatment goal (table 3 ).

\section{WHO/ISH 2003 guideline}

In contrast to JNC7, the WHO/ISH 2003 guideline, published shortly after the JNC7 guideline, recommends antihypertensive medication in persons with $\mathrm{SBP} \geq 140 \mathrm{~mm}$ $\mathrm{Hg}$ or DBP $\geq 90 \mathrm{~mm} \mathrm{Hg}$ exhibiting high cardiovascular disease risk defined by the presence of cardiovascular risk factors, target-organ damage or hypertension-associated clinical conditions (online supplemental figure S2). We defined extensive atherosclerotic plaque as a sign of target-organ damage by ankle-brachial index $(\mathrm{ABI})<0.9$ or CIMT $>0.9 \mathrm{~mm}$, as suggested by the ESH/ESC 2013 guideline. Antihypertensive medication is only recommended in all persons when they exhibit $\mathrm{SBP} \geq 180 \mathrm{~mm} \mathrm{Hg}$ or DBP $\geq 110 \mathrm{~mm} \mathrm{Hg}$ (online supplemental figure S2).

The WHO/ISH 2003 guideline recommended a much lower percentage of HNR participants hitherto not taking antihypertensive medication for antihypertensive medication compared with the JNC7 guideline (20.3\% vs $37.2 \%$, table 2). When CIMT was excluded from the 
Table 1 Baseline characteristics of the total Heinz Nixdorf Recall study cohort stratified by antihypertensive medication intake at the baseline examination

\section{Baseline characteristics}

Age, years

$>55 />60$ years for males/females, $n(\%)$

$>65$ years, $n(\%)$

Male sex, $\mathrm{n}(\%)$

Systolic blood pressure, $\mathrm{mm} \mathrm{Hg}$

Diastolic blood pressure, $\mathrm{mm} \mathrm{Hg}$

Total cholesterol, $\mathrm{mg} / \mathrm{dL}$

Total cholesterol $>310 \mathrm{mg} / \mathrm{dL}, \mathrm{n}(\%)$

Total cholesterol $>240 \mathrm{mg} / \mathrm{dL}$ or LDL cholesterol $>160 \mathrm{mg} / \mathrm{dL}$, $\mathrm{n}(\%)$

LDL cholesterol, $\mathrm{mg} / \mathrm{dL}$

$\mathrm{HDL}$ cholesterol, $\mathrm{mg} / \mathrm{dL}$

$\mathrm{HDL}<40 / 45 \mathrm{mg} / \mathrm{dL}$ for males/females, $\mathrm{n}(\%)$

Triglycerides, $\mathrm{mg} / \mathrm{dL}$, median (IQR)

LDL cholesterol $>100 \mathrm{mg} / \mathrm{dL}$ or triglycerides $>150 \mathrm{mg} / \mathrm{dL}, \mathrm{n}(\%)$

Glucose, mg/dL

Diabetes mellitus, $\mathrm{n}(\%)$

Serum creatinine, $\mathrm{mg} / \mathrm{dL}$

GFR, $\mathrm{mL} / \mathrm{min} / 1.73 \mathrm{~m}^{2}$

CKD, $n(\%)$

Smoking, $\mathrm{n}(\%)$

BMI, $\mathrm{kg} / \mathrm{m}^{2}$

Overweight, $\mathrm{n}(\%)$

Physical inactivity, $\mathrm{n}(\%)$

Overweight or physical inactivity, n (\%)

CIMT, mm

CIMT>0.9mm, n (\%)

$\mathrm{ABI}$

$\mathrm{ABI}<0.9, \mathrm{n}(\%)$

Left ventricular hypertrophy on ECG, $n$ (\%)

Atrial fibrillation on ECG, $\mathrm{n}(\%)$

Stroke history, n (\%)

CHD history, $\mathrm{n}(\%)$

Heart failure history, $\mathrm{n}(\%)$

Peripheral artery disease history, n (\%)

$\mathrm{Ml}$ history in first-degree relatives, $\mathrm{n}(\%)$

Early-onset menopause, n (\%)

Antidiabetic medications, $\mathrm{n}(\%)$

ASCVD score, \%, median (IQR)

SCORE, \%, median (IQR)

Cardiovascular event, n (\%)
Not taking

antihypertensive medication $(\mathrm{n}=3092)$

$58.1 \pm 7.5$

$1189(38.4)$

$672(21.7)$

$1509(48.8)$

$130.6 \pm 20.3$

$81.1 \pm 10.6$

$230.9 \pm 38.9$

$81(2.6)$

$1319(42.8)$

$146.7 \pm 36.5$

$59.8 \pm 17.4$

$384(12.5)$

$116.0(84.3-166.0)$

2845 (92.6)

$107.9 \pm 23.2$

267 (8.6)

$0.91 \pm 0.22$

$81.3 \pm 18.4$

$190(6.2)$

815 (26.3)

$27.1 \pm 4.2$

$649(21.1)$

1429 (46.1)

1737 (56.1)

$0.66 \pm 0.13$

$110(4.5)$

$1.14 \pm 0.14$

98 (3.2)

58 (1.9)

$21(0.68)$

43 (1.4)

$49(1.6)$

36 (1.2)

41 (1.3)

$773(27.4)$

$290(9.4)$

$90(3.1)$

$6.5(3.0-13.2)$

$3.0(1.0-7.0)$

$52(1.7)$

\section{Taking antihypertensive}

medication $(n=1691) \quad P$ value

$62.4 \pm 7.5$

$1015(60.0)$

735 (43.7)

$873(51.6)$

$137.7 \pm 21.0$

$82.0 \pm 11.0$

$226.0 \pm 39.4$

$42(2.5)$

668 (39.6)

$143.5 \pm 35.5$

0.004

$54.7 \pm 16.4$

$<0.001$

342 (20.2)

$<0.001$

141.0 (101.0-200.0)

$<0.001$

1571 (93.5)

0.143

$118.1 \pm 34.7$

$<0.001$

386 (22.8)

$<0.001$

$0.97 \pm 0.27$

$<0.001$

$76.5 \pm 18.2$

$<0.001$

231 (13.7)

$<0.001$

307 (18.1)

$<0.001$

$29.4 \pm 5.0$

$<0.001$

670 (39.7)

$<0.001$

921 (54.5)

$<0.001$

$1200(70.7)$

$<0.001$

$0.71 \pm 0.13$

$<0.001$

107 (7.9)

$<0.001$

$1.10 \pm 0.17$

$<0.001$

156 (9.4)

$<0.001$

$70(4.2)$

$<0.001$

56 (3.31)

$<0.001$

88 (5.2)

$<0.001$

$262(15.4)$

$<0.001$

$127(7.7)$

$<0.001$

64 (3.9)

$<0.001$

454 (28.4)

0.473

162 (9.7)

0.796

175 (10.7)

$<0.001$

13.9 (7.2-24.3)

$<0.001$

$5.0(3.0-10.0)$

$<0.001$

$69(4.1)$

$<0.001$

Unless stated otherwise, values are presented as mean \pm SD.

$\mathrm{ABI}$, ankle-brachial index; ASCVD, atherosclerotic cardiovascular disease; BMI, body mass index; CHD, coronary heart disease;

CIMT, common-carotid artery intima-media thickness; CKD, chronic kidney disease; GFR, glomerular filtration rate; HDL, high-density lipoprotein cholesterol; LDL, low-density lipoprotein cholestero; MI, myocardial infarction; SCORE, Systematic COronary Risk Evaluation score. 
Table 2 Prevalence of antihypertensive medication recommendations according to the different hypertension management guidelines in the Heinz Nixdorf Recall cohort

\begin{tabular}{|c|c|c|}
\hline & $\begin{array}{l}\text { Threshold for initiating antihypertensive medication } \\
\text { treatment }\end{array}$ & $\begin{array}{l}\text { Antihypertensive medication recommendation } \\
\text { among participants hitherto not taking } \\
\text { antihypertensive medication*, n }(\%, 95 \% \mathrm{Cl})\end{array}$ \\
\hline JNC7 2003 & $\begin{array}{l}\geq 140 / 90 \mathrm{~mm} \mathrm{Hg} \text { in all and } \geq 130 / 80 \mathrm{~mm} \mathrm{Hg} \text { in high } \\
\text { cardiovascular risk patients }\end{array}$ & $1146(37.2,35.5$ to 38.9$)$ \\
\hline WHO/ISH 2003 & $\begin{array}{l}\geq 180 / 110 \mathrm{~mm} \mathrm{Hg} \text { in all and } \geq 140 / 90 \mathrm{~mm} \mathrm{Hg} \text { in high } \\
\text { cardiovascular risk patients }\end{array}$ & $601(20.3,18.9$ to 21.8$)$ \\
\hline ESH/ESC 2013 & $\begin{array}{l}\geq 160 / 100 \mathrm{~mm} \mathrm{Hg} \text { in all and } \geq 140 / 90 \mathrm{~mm} \mathrm{Hg} \text { in high } \\
\text { cardiovascular risk patients }\end{array}$ & $523(17.8,16.4$ to 19.2$)$ \\
\hline ACC/AHA 2017 & $\begin{array}{l}\geq 140 / 90 \mathrm{~mm} \mathrm{Hg} \text { in all and } \geq 130 / 80 \mathrm{~mm} \mathrm{Hg} \text { in high } \\
\text { cardiovascular risk patients }\end{array}$ & $1418(45.8,44.0$ to 47.6$)$ \\
\hline ESC/ESH 2018 & $\begin{array}{l}\geq 160 / 100 \mathrm{~mm} \mathrm{Hg} \text { in all and } \geq 140 / 90 \mathrm{~mm} \mathrm{Hg} \text { in high } \\
\text { cardiovascular risk patients }\end{array}$ & $821(26.7,25.1$ to 28.3$)$ \\
\hline ISH 2020 & $\begin{array}{l}\geq 160 / 100 \mathrm{~mm} \mathrm{Hg} \text { in all and } \geq 140 / 90 \mathrm{~mm} \mathrm{Hg} \text { in high } \\
\text { cardiovascular risk patients }\end{array}$ & $772(25.0,23.5$ to 26.5$)$ \\
\hline
\end{tabular}

${ }^{*} \mathrm{n}=3092$.

ACC, American College of Cardiology; AHA, American Heart Association; ESC, European Society of Cardiology; ESH, European Society of Hypertension; ISH, International Society of Hypertension; ; JNC7, Joint National Committee on Prevention, Detection, Evaluation, and Treatment of High Blood Pressure 7.

antihypertensive medication recommendation in sensitivity analyses due to $4.3 \%$ of missing data (online supplemental table S3), results were very similar (antihypertensive medication recommended in $\mathrm{n}=581$, that is, $19.0 \%, 95 \%$ CI $17.6 \%$ to $20.4 \%$ ). The treatment goal for antihypertensive medication of SBP $<140 \mathrm{~mm} \mathrm{Hg}$ and DBP $<90 \mathrm{~mm} \mathrm{Hg}$ (online supplemental table S2) was not achieved in $48.1 \%$ of HNR participants taking antihypertensive medication (table 3).

\section{ESH/ESC 2013 guideline}

Consistent with the WHO/ISH 2003 guideline, and in contrast to the JNC7 guideline, the ESH/ESC 2013 guideline recommends antihypertensive medication in persons with SBP $\geq 140 \mathrm{~mm} \mathrm{Hg}$ or DBP $\geq 90 \mathrm{~mm} \mathrm{Hg}$ exhibiting high cardiovascular disease risk (online supplemental figure
S3), which is defined by asymptomatic organ damage, diabetes mellitus, established cardiovascular disease or renal disease (legend to online supplemental figure S3). In contrast to the WHO/ISH 2003 guideline, antihypertensive medication is recommended in all persons at a lower BP level (SBP $\geq 160 \mathrm{~mm} \mathrm{Hg}$ or DBP $\geq 100 \mathrm{~mm} \mathrm{Hg}$ (online supplemental figure S3)).

According to the ESH/ESC 2013 guideline, 17.8\% of participants hitherto not taking antihypertensive medication were recommended for antihypertensive medication (table 2), which is similar to the WHO/ISH 2003 and much below JNC7 guidelines.

When CIMT was excluded from the antihypertensive medication recommendation in sensitivity analyses due to $4.8 \%$ of missing data (online supplemental table S3),

Table 3 Prevalence of blood pressure above treatment goal according to the different hypertension management guidelines in the Heinz Nixdorf Recall cohort

\begin{tabular}{|c|c|c|}
\hline & $\begin{array}{l}\text { Treatment targets for antihypertensive } \\
\text { medication treatment }\end{array}$ & $\begin{array}{l}\text { Blood pressure above treatment goal among } \\
\text { participants already taking antihypertensive } \\
\text { medication*, } \mathrm{n}(\%, 95 \% \mathrm{Cl})\end{array}$ \\
\hline JNC7 2003 & $\begin{array}{l}<140 / 90 \mathrm{~mm} \mathrm{Hg} \text { in all and }<140 / 80 \mathrm{~mm} \mathrm{Hg} \text { in high } \\
\text { cardiovascular risk patients }\end{array}$ & $949(56.4,54.0$ to 58.8$)$ \\
\hline WHO/ISH 2003 & $<140 / 90 \mathrm{~mm} \mathrm{Hg}$ in all patients & $813(48.1,45.7$ to 50.5$)$ \\
\hline ESH/ESC 2013 & $\begin{array}{l}<140 / 90 \mathrm{~mm} \mathrm{Hg} \text { in all and }<140 / 85 \mathrm{~mm} \mathrm{Hg} \text { in } \\
\text { patients with diabetes }\end{array}$ & $830(49.1,46.7$ to 51.5$)$ \\
\hline ESC/ESH 2018 & $<140 / 90 \mathrm{~mm} \mathrm{Hg}$ in all patients & $813(48.1,45.7$ to 50.5$)$ \\
\hline ISH 2020 & $<140 / 90 \mathrm{~mm} \mathrm{Hg}$ in all patients & $813(48.1,45.7$ to 50.5$)$ \\
\hline
\end{tabular}

${ }^{*} \mathrm{n}=1691$.

ACC, American College of Cardiology; AHA, American Heart Association; ESC, European Society of Cardiology; ESH, European Society of Hypertension; JNC7, Joint National Committee on Prevention, Detection, Evaluation, and Treatment of High Blood Pressure 7; ISH, International Society of Hypertension.; 
results were very similar (antihypertensive medication recommended in $\mathrm{n}=497$, ie, $16.3 \%, 95 \%$ CI $15.0 \%$ to $17.6 \%)$. For antihypertensive medication, the ESH/ESC 2013 guideline suggests a treatment goal of SBP $<140 \mathrm{~mm}$ $\mathrm{Hg}$ and $\mathrm{DBP}<90 \mathrm{~mm} \mathrm{Hg}$ in all persons, and a treatment goal of SBP $<140 \mathrm{~mm} \mathrm{Hg}$ and $\mathrm{DBP}<85 \mathrm{~mm} \mathrm{Hg}$ in persons with diabetes mellitus (online supplemental table S2), which was not achieved in $49.1 \%$ of HNR participants taking antihypertensive medication (table 3 ).

\section{ACC/AHA 2017 guideline}

Like the JNC7 guideline, its update, the ACC/AHA 2017 guideline, recommends antihypertensive medication in persons with $\mathrm{SBP} \geq 130 \mathrm{~mm} \mathrm{Hg}$ or $\mathrm{DBP} \geq 80 \mathrm{~mm} \mathrm{Hg}$ exhibiting high cardiovascular disease risk (online supplemental figure S4). While in the JNC7 guideline, high cardiovascular disease risk was defined as the presence of diabetes mellitus or CKD, the ACC/AHA 2017 guideline additionally includes patients with peripheral artery disease, a history of cardiovascular disease, and $\geq 10 \%$ 10 year atherosclerotic cardiovascular disease (ASCVD) risk in their definition of high cardiovascular disease risk (legend to online supplemental figure S4). As in the JNC7 guideline, antihypertensive medication is recommended in all persons with $\mathrm{SBP} \geq 140 \mathrm{~mm} \mathrm{Hg}$ or $\mathrm{DBP} \geq 90 \mathrm{~mm} \mathrm{Hg}$ (online supplemental figure $\mathrm{S} 4$ ).

Based on the ACC/AHA 2017 guideline, an increased percentage of HNR participants hitherto not taking antihypertensive medication was recommended for antihypertensive medication $(45.8 \%$, table 2$)$. In contrast to all previous guidelines, the ACC/AHA 2017 guideline suggests a lower treatment $\mathrm{BP}$ goal of $\mathrm{SBP}<130 \mathrm{~mm} \mathrm{Hg}$ and DBP $<80 \mathrm{~mm} \mathrm{Hg}$ in all patients and of SBP $<130$ in non-institutionalised, ambulatory, community-living adults $\geq 65$ years (online supplemental table S2), which was not achieved in $69.7 \%$ of HNR participants taking antihypertensive medication (table 3 ).

\section{ESC/ESH 2018 guideline}

As already in the previous European guideline, but contrary to the American guidelines, ESC/ESH 2018 recommends antihypertensive medication in all persons with $\mathrm{SBP} \geq 140 \mathrm{~mm} \mathrm{Hg}$ or DBP $\geq 90 \mathrm{~mm} \mathrm{Hg}$ exhibiting high cardiovascular disease risk (online supplemental figure S5), which is defined using Systematic COronary Risk Evaluation (SCORE) and cardiovascular disease imaging parameters (legend to online supplemental figure S5).

Based on the latest ESC/ESH hypertension management guideline, $26.7 \%$ of HNR participants hitherto not taking antihypertensive medication were recommended for antihypertensive medication, which is higher than ESC/ESH 2013 and WHO/ISH 2003 guidelines and lower than the American guidelines (table 2). BP treatment goals are not precisely defined in the ESC/ESH 2018 guideline, rather an overview of literature including different patient subsets is given to offer information for practitioners to be able to decide on an individual patient basis. In general, a treatment goal of SBP $<140 \mathrm{~mm} \mathrm{Hg}$ and DBP $<90 \mathrm{~mm} \mathrm{Hg}$ is suggested for all persons (online supplemental table S2). With this treatment goal, $48.1 \%$ of HNR participants taking antihypertensive medication exhibited BP values above the treatment goal (table 3). When in a sensitivity analysis a treatment goal of SBP $<130 \mathrm{~mm} \mathrm{Hg}$ and $\mathrm{DBP}<80 \mathrm{~mm} \mathrm{Hg}$ is defined in all persons $<65$ years and a treatment goal of SBP $<140 \mathrm{~mm} \mathrm{Hg}$ and DBP $<90 \mathrm{~mm} \mathrm{Hg}$ in all persons $\geq 65$ years, $1054 \mathrm{HNR}$ participants taking antihypertensive medication $(62.3 \%$, $60.0 \%$ to $64.7 \%$ ) exhibited BP above treatment goal.

\section{ISH 2020 guideline}

As already suggested in the current and previous European guideline and in WHO/ISH 2003, but contrary to the American guidelines, ISH 2020 recommends antihypertensive medication in all persons with SBP $\geq 140 \mathrm{~mm}$ $\mathrm{Hg}$ or DBP $\geq 90 \mathrm{~mm} \mathrm{Hg}$ exhibiting high cardiovascular disease risk (online supplemental figure S6), which is defined by the number of cardiovascular risk factors as well as hypertension-mediated organ damage and diseases (legend to online supplemental figure S6). Similar to the current and previous European guideline but in contrast with the American guidelines and WHO/ISH 2003 (suggesting a lower and higher threshold, respectively), antihypertensive medication is recommended in all persons indepent from cardiovascular risk for SBP $\geq 160 \mathrm{~mm} \mathrm{Hg}$ or DBP $\geq 100 \mathrm{~mm} \mathrm{Hg}$ (online supplemental figure S6).

Based on the most recent ISH 2020 hypertension management guideline, $25.0 \%$ of HNR participants hitherto not taking antihypertensive medication were recommended for antihypertensive medication, which is similar to ESC/ESH 2018, higher than WHO/ISH 2003 and ESH/ESC 2013 and lower than the American guidelines (table 2). An essential treatment goal of SBP $<140 \mathrm{~mm} \mathrm{Hg}$ and DBP $<90 \mathrm{~mm} \mathrm{Hg}$ is suggested for all persons (online supplemental table S2). With this treatment goal, $48.1 \%$ of HNR participants taking antihypertensive medication exhibited BP values above the treatment goal (table 3).

\section{Differences in baseline cardiovascular risk factors between HNR participants with and without an antihypertensive medication recommendation according to the different hypertension management guidelines}

Comparing participants with and without an antihypertensive medication recommendation according to the JNC7 guideline, participants with an antihypertensive medication recommendation expectedly were older, exhibited higher BP, and were more likely to suffer from diabetes mellitus or $\mathrm{CKD}$, since those variables are included in the definition of treatment recommendation (legend to online supplemental figure S1). Additionally, participants with an antihypertensive medication recommendation were more likely to be male, smokers, overweight or physically inactive. They more frequently exhibited hyperlipoproteinaemia, left ventricular hypertrophy (LVH), and had a history of vascular disease, and higher ASCVD score and SCORE than participants 
without an antihypertensive medication recommendation (online supplemental table S4). Interestingly, the majority of factors not included in the JNC7 antihypertensive medication recommendation, which were more present in HNR participants recommended for antihypertensive medication than in participants not recommended for antihypertensive medication, were included in later guidelines, such as the WHO/ISH 2003, which included sex, overweight, CIMT, ABI and LVH in addition to the variables included in the JNC7 guideline. Also, the former 2013 and recent 2018 European guideline include $\mathrm{ABI}$ as a marker for subclinical atherosclerosis into their antihypertensive medication recommendation, while CIMT is only included in the former 2013 guideline but removed from the recent 2018 version due to class III recommendation for CIMT in the assessment of cardiovascular risk. ${ }^{16}$ The most recent ISH 2020 guideline however makes no specific suggestion about the assessment of these subclinical atherosclerosis markers. Not only in the JNC7 but also in all subsequent guidelines, participants with an antihypertensive medication recommendation exhibited a more pronounced cardiovascular risk profile than participants without a recommendation (online supplemental tables S4-S9).
Differences in incident cardiovascular events during the 5-year follow-up between HNR participants with and without an antihypertensive medication recommendation according to the different hypertension management guidelines

Participants with an antihypertensive medication recommendation based on the recent American hypertension management guideline (ACC/AHA 2017) had an elevated incidence of cardiovascular events compared with participants without a recommendation $(2.5 \%, 95 \%$ CI $1.7 \%$ to $3.3 \%$ vs $1.1 \%, 95 \%$ CI $0.6 \%$ to $1.7 \%$, figure 1 , online supplemental table S7). For all other guidelines, which recommended antihypertensive medication in fewer persons than the recent American guideline, the incidence of cardiovascular events did not significantly differ between participants with and without an antihypertensive medication recommendation (figure 1, online supplemental tables S4-S6, S8, S9). After adjustment for age and sex, however, the ACC/AHA 2017 guideline discriminated cardiovascular event incidence only marginally better than the other guidelines, especially compared with the ESH/ESC 2013 guideline (figure 1). The age-adjusted and sex-adjusted incidence rates show that the higher risk in participants with an antihypertensive medication recommendation based on ACC/

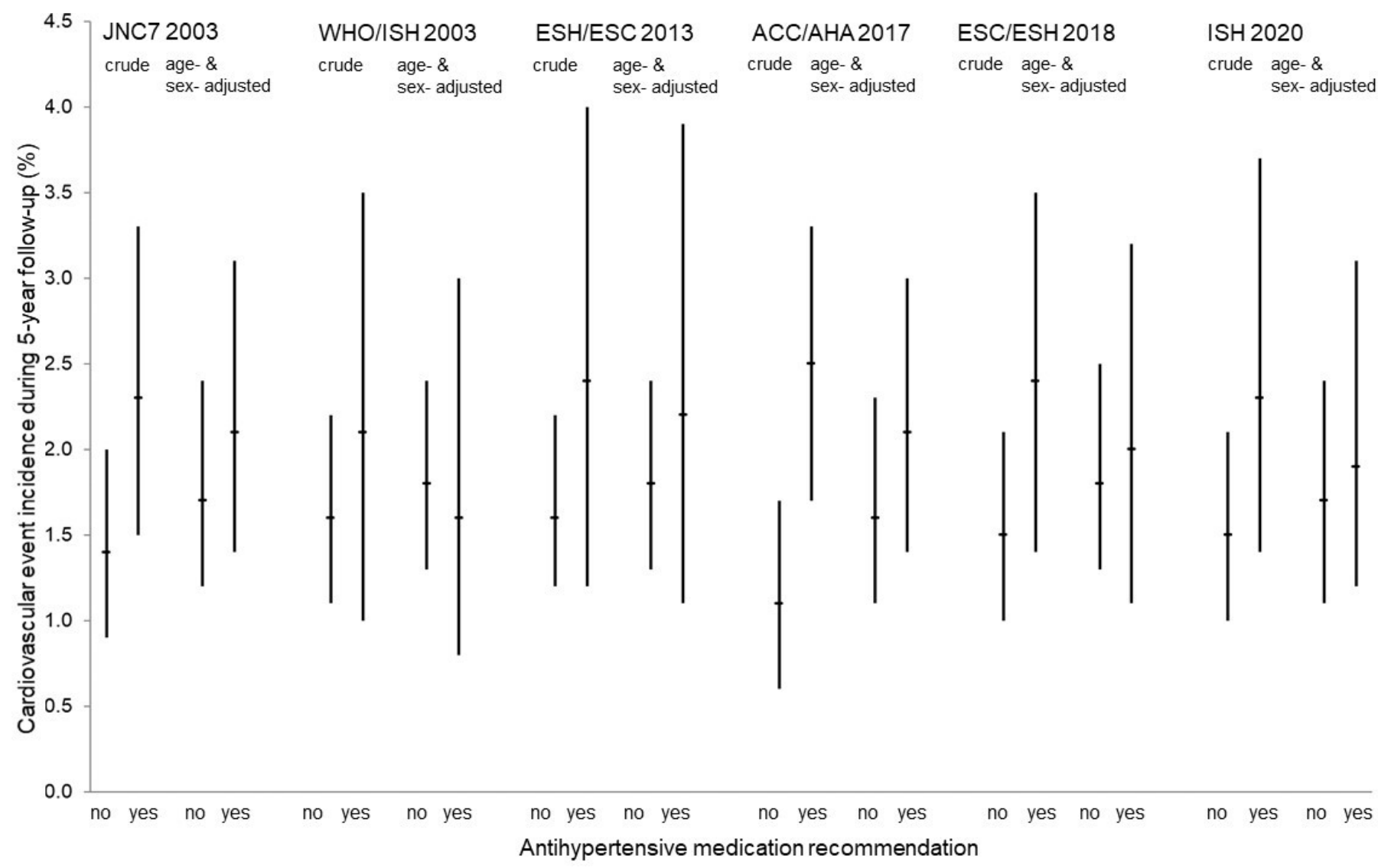

Figure 1 Crude and age-adjusted and sex-adjusted cardiovascular event incidence during the 5-year follow-up stratified by antihypertensive medication recommendation at the baseline examination according to the different hypertension management guidelines. ACC, American College of Cardiology; AHA, American Heart Association; ESC, European Society of Cardiology; ESH, European Society of Hypertension; JNC7, Joint National Committee on Prevention, Detection, Evaluation, and Treatment of High Blood Pressure 7; ISH, International Society of Hypertension. 
AHA 2017 (2.1\%, 95\% CI 1.4\% to 3.0\%) compared with participants without a recommendation $(1.6 \%, 95 \%$ CI $1.1 \%$ to $2.3 \%$ ) is to a considerable degree based on nonmodifiable risk factors.

\section{DISCUSSION}

When applying six different hypertension management guidelines published by American, European and international organisations to the HNR cohort, we observed that the current ACC/AHA guideline recommended a higher percentage of participants hitherto not taking antihypertensive medication for antihypertensive medication $(45.8 \%)$ compared with the JNC7 $(37.2 \%)$, ESH/ ESC 2013 (17.8\%), ESC/ESH 2018 (26.7\%), WHO/ISH $2003(20.3 \%)$ or ISH 2020 (25.0\%) guidelines. Additionally, a higher proportion of participants hitherto taking antihypertensive medication still had a BP above treatment goal $(69.7 \%)$ compared with the JNC7 (56.4\%), ESH/ESC 2013 (49.1\%), and ESC/ESH 2018, WHO/ISH 2003, or ISH 2020 (all 48.1\%) guidelines.

Consistent with the idea of reducing future cardiovascular disease via stricter antihypertensive medication recommendations, participants with an antihypertensive medication recommendation according to the ACC/ AHA 2017 guideline experienced significantly more cardiovascular events during the 5-year follow-up (2.5\%) compared with participants without a recommendation $(1.1 \%, p=0.003)$. For all other guidelines, an antihypertensive medication recommendation was not significantly associated with incident cardiovascular events.

Also, in a previous comparison of the ACC/AHA 2017 with ESH/ESC 2013 guidelines within the Swiss population-based CoLaus/PsyCoLaus study, the ACC/ AHA 2017 guideline resulted in a more frequent antihypertensive medication recommendation $(40.3 \%$ vs $31.3 \%$ ). Similarly, BP above treatment goals was more frequent according to the ACC/AHA 2017 (53.8\%) compared with the ESH/ESC 2013 (38.2\%) guideline. ${ }^{17}$ When the ACC/AHA 2017 guideline was compared with the JNC7 guideline within the American population-based National Health and Nutrition Examination Survey, an antihypertensive medication recommendation, in contrast to our analyses, was not increased substantially (36.2\% vs $34.3 \%)$. However, similar to our analyses, BP above treatment goal in persons taking antihypertensive medication was strongly increased (53.4\% vs $39.0 \%)$.

Whereas our analyses and those of the Swiss ${ }^{17}$ and American ${ }^{4}$ populations consistently showed that application of the ACC/AHA 2017 guideline would lead to increased prescription of antihypertensive medication, we, for the first time, revealed that participants who hitherto were not taking antihypertensive medication, but who would be recommended for antihypertensive medication based on the ACC/AHA 2017 guideline, had a higher incidence of cardiovascular events than participants without a recommendation. The increased cardiovascular event risk in participants with an antihypertensive medication recommendation was, however, considerably based on the non-modifiable risk factors age and sex. Further, it has to be considered that the ACC/AHA 2017 guideline recommended the highest proportion of participants for antihypertensive medication; thus, a higher incidence of cardiovascular events is expected here since even in highnormal BP the incidence rate is about $1 \%$ per year. $^{18}$

It has to be kept in mind that pharmacological antihypertensive therapy can be associated with side effects, ${ }^{19}$ and therefore does not necessarily result in reduced cardiovascular risk. In a Cochrane Review including randomised controlled trials (RCTs) of at least 1-year duration, which analysed the effect of pharmacological therapy on cardiovascular events in hypertensive patients with SBP $140-159 \mathrm{~mm} \mathrm{Hg}$ or DBP 90-99 mm Hg, antihypertensive medication did not significantly reduce cardiovascular events, but significantly increased withdrawals due to adverse effects compared with placebo. Thus, the Cochrane Review concluded that more RCTs are needed to evaluate whether the benefits of antihypertensive medication exceed the harms in patients with SBP/DBP $<160 / 100 \mathrm{~mm} \mathrm{Hg}^{20}$

Whereas we could highlight the differences in hypertension guidelines and the resulting implications on the population level, a key question remains why guidelines themselves differ. Possible reasons might include differences in the development process, procedural and decision policies as well as the definitions of level of evidence and strength of recommendations. Particularly in the case of ACC/AHA 2017 and ESC/ESH 2018, which were published roughly at the same time, guideline authors have presumably had access to the same evidence-base.

The ESC/ESH 2018 guideline was developed based on a fixed procedure representing the basis for the development of all published ESC Clinical Practice Guidelines. ${ }^{21}$ Guideline development policies of ACC/AHA 2017 are published in the ACC/AHA guideline methodology manual $^{22}$ but are kept more flexible to allow innovative concepts like the modular knowledge chunk format. Another difference is that formal systematic review is only performed for questions deemed of utmost clinical importance (concerns BP treatment goals of the present paper).

Data used for the development of hypertension guidelines differs in the level of evidence and strength of recommendation. Both ESC/ESH 2018 and ACC/AHA 2017 present level of evidence and strength of recommendation for their recommendations; however, there are slight differences in the definition of level of evidence. ESC/ ESH 2018 defines level of evidence $\mathrm{A}$ as data derived from multiple RCTs or meta-analyses, and level of evidence B as data derived from a single RCT or large non-randomised studies. ACC/AHA 2017 defines level of evidence A as high-quality evidence (where the method of quality assessment is described as evolving and not concretely specified) from >1 RCT, meta-analyses of high-quality RCTs, and $\geq 1$ RCTs corroborated by high-quality registry studies. Level of evidence B is split into randomised and 
non-randomised with non-randomised being similar to the ESC/ESH 2018 definition. Randomised differs from the ESC/ESH 2018 definition in that it includes moderate-quality evidence from $\geq 1$ RCTs or meta-analyses of moderate-quality RCTs. Level of evidence C is similar except that ACC/AHA 2017 separates expert opinion from limited data and describes limited data in more detail. Regarding classes of evidence, ACC/AHA 2017 quantifies the benefit risk relationship and splits class 3 ('not recommended' in ESC/ESH 2018) into 'no benefit' and 'harm'.

Further aspects relate to the evidence used for the guideline recommendations regarding $\mathrm{BP}$ classification, antihypertensive treatment and BP treatment goal. The ESC/ESH 2018 recommendation to classify BP as optimal, normal, high-normal and grade 1-3 hypertension according to office BP (online supplemental figure S5) is a class 1 recommendation with a level of evidence C. The recommendation to define hypertension as office $\mathrm{BP} \geq 140 / 90 \mathrm{~mm} \mathrm{Hg}$ does not differ from previous ESC/ ESH guidelines and is based on evidence from multiple RCTs that treatment of patients with these BP values is beneficial. The ACC/AHA 2017 recommendation to classify BP as normal, elevated, and grade1 and 2 hypertension according to office BP (online supplemental figure S4) is a class 1 recommendation with a level of evidence $\mathrm{B}$ non-randomised. This categorisation differs from JNC 7, with grade 1 hypertension now defined as BP 130-139/80-89 mm Hg, and with grade 2 hypertension corresponding to grade 1 and 2 hypertension in JNC 7 . The rationale for this categorisation is highly influenced by observational data showing gradient progressively increasing cardiovascular risk from normal to grade 2 hypertension and by the idea to increase alterness of the negative consequences of already slightly elevated $\mathrm{BP}$ and thus increase lifestyle modification interventions.

Regarding antihyperensive medication recommendation, the ESC/ESH 2018 recommendation to treat all patients with grade 2 and 3 hypertension independent of cardiovascular risk is a class 1 recommendation with level of evidence A. Also the recommendation to treat grade 1 hypertension at high cardiovascular risk is a class 1 recommendation level of evidence A. Recommendations are based on RCTs and reviews/meta-analyses of RCTs. ${ }^{23-26}$ Even though the evidence given argues in favour of treating already BP $<140 / 90 \mathrm{~mm} \mathrm{Hg}$, there is a lower number of studies investigating treatment benefits in $\mathrm{BP}$ $<140 / 90 \mathrm{~mm} \mathrm{Hg}$ than in $\mathrm{BP} \geq 140 / 90 \mathrm{~mm} \mathrm{Hg}$, which led ESC/ESH keep the higher treatment threshold compared with ACC/AHA 2017. The ACC/AHA 2017 antihypertensive medication recommendations to treat patients with BP 130-139/80-89 in case of high cardiovascular risk is a class 1 recommendation with level of evidence A for SBP and level of evidence $\mathrm{C}$ (expert opinion) for DBP. The recommendation to treat all patients with $\mathrm{BP} \geq 140 / 90 \mathrm{~mm}$ $\mathrm{Hg}$ is also a class 1 recommendation with level of evidence $\mathrm{C}$ (limited data). Even though there is considerable overlap in the evidence base for the ESC/ESH 2018 and
ACC/AHA 2017 antihypertensive medication recommendations, ${ }^{23} 2526$ ACC/AHA 2017 but not ESC/ESH 2018 regarded the available evidence as sufficient to reduce the treatment threshold. Further, the ACC/AHA 2017 antihypertensive medication recommendations were strongly influenced by the Systolic Blood Pressure Intervention Trial (SPRINT) results, ${ }^{27}$ whereas ESC/ESH 2018 does not regard the SPRINT results as adequate to support the decision to initiate antihypertensive treatment since most SPRINT participants were already treated at baseline. As disclosed in ACC/AHA 2017, three of the authors had leadership roles in SPRINT but did not chair committee discussions in which the SPRINT results were considered. SPRINT is a multicentre RCT comparing the standard SBP target of $<140 \mathrm{~mm} \mathrm{Hg}$ to a more intensive target of $<120 \mathrm{~mm}$ Hg. Recruitment focused on volunteers $\geq 50$ years with an average baseline $\mathrm{SBP} \geq 130 \mathrm{~mm} \mathrm{Hg}$ (mean BP $140 / 78 \mathrm{~mm} \mathrm{Hg}$ ) and evidence of cardiovascular disease, CKD, and 10-year Framingham cardiovascular disease risk score $\geq 15 \%$. The primary outcome included a myocardial infarction, acute coronary syndrome, stroke, heart failure, or cardiovascular disease death. ${ }^{27}$ After 3 years of follow-up, participants assigned to the intensive treatment target had a significantly lower risk of the primary outcome than participants assigned to the standard treatment goal, even in a subgroup analysis of participants $\geq 75$ years. However, serious adverse events of hypotension, syncope, electrolyte abnormalities, and acute kidney injury or failure, and injurious falls (only in participants $\geq 75$ years), occurred more frequently in the intensive than in the standard treatment target group. ${ }^{28}{ }^{29}$ Whereas ACC/AHA 2017 treatment recommendations for individuals with high cardiovascular risk are strongly influenced by SPRINT, recommendations for individuals with intermediate risk are partly influenced by the Heart Outcomes Prevention Evaluation-3 (HOPE-3) trial. The goal of HOPE-3 was to assess the safety and efficacy of cholesterol lowering, BP lowering, or both in patients exhibiting $\mathrm{SBP}<160 \mathrm{~mm} \mathrm{Hg}$ with an intermediate CVD risk $(\sim 1 \%$ annually) without known cardiovascular disease. Twelve thousand seven hundred five patients (mean age 65.8 years, $46 \%$ female, mean BP $138 / 82 \mathrm{~mm} \mathrm{Hg}$, median two cardiovascular risk factors) were randomised in a $2 \times 2$ factorial design to either (1) cholesterol lowering with rosuvastatin $10 \mathrm{mg}(\mathrm{n}=6361)$ or placebo $(\mathrm{n}=6344)$, (2) BP lowering with candesartan $16 \mathrm{mg}$ +hydrochlorothiazide $(\mathrm{n}=6356)$ or placebo $(\mathrm{n}=6349)$, (3) cholesterol and BP lowering $(n=3180)$ or placebo $(n=3168)$. Contrary to the results of SPRINT in patients with high cardiovascular risk, the total cohort of patients with intermediate cardiovascular risk did not benefit from BP lowering (mean decrease in $\mathrm{SBP} / \mathrm{DBP}=6 / 3 \mathrm{~mm} \mathrm{Hg}$ ) regarding longterm cardiovascular risk reduction (5.6 years follow-up) compared with placebo. Only cholesterol lowering alone and in combination with $\mathrm{BP}$ lowering was superior to placebo in reducing long-term cardiovascular risk. In the total cohort, symptomatic hypotension was significantly more frequent in the active BP lowering arm than in the 
placebo arm. However, there was a significant interaction with baseline SBP showing that patients in the subgroup of the upper third of SBP (>143.5 mm Hg) had a significant reduction in cardiovascular risk in the active arm compared with placebo, which matches the suggestion of the JNC7 and ACC/AHA 2017 guideline to use antihypertensive medication only in intermediate cardiovascular risk patients exhibiting BP $>140 / 90 \mathrm{~mm} \mathrm{Hg} .{ }^{24} 3031$ There are important differences between the SPRINT and HOPE-3 trial to consider: HOPE-3 did not enrol patients based on baseline values of cholesterol or BP, but based on their baseline risk for cardiovascular events. HOPE-3 enrolled patients from many different countries, making results more broadly generalisable. BP was lowered less intensively in HOPE-3, on average SBP was still $>130 \mathrm{~mm}$ $\mathrm{Hg}$ whereas in SPRINT it was lowered to $<120 \mathrm{~mm} \mathrm{Hg}$ in the intensive treatment arm. In HOPE-3, it remains unclear if the use of other antihypertensive agents would have demonstrated a benefit.

Regarding treatment goals, ESC/ESH 2018 did not define concrete goals but rather give an overview of literature regarding different treatment goals for a variety of comorbidities to facilitate individual patient decisions. ACC/AHA 2017 in contrast suggested a BP treatment goal of $<130 / 80 \mathrm{~mm} \mathrm{Hg}$ independent of comorbidities but it has to be considered that this treatment goal is a class 1 recommendation only for high cardiovascular risk patients (level of evidence B (randomised) for SBP and level of evidence $\mathrm{C}$ (expert opinion) for DBP). The BP treatment goal of $<130 / 80 \mathrm{~mm} \mathrm{Hg}$ for patients without increased cardiovascular risk only represents a class $2 \mathrm{~b}$ recommendation with level of evidence B (nonrandomised) for SBP and level of evidence $\mathrm{C}$ (expert opinion) for DBP.

\section{Strengths and limitations of the study}

Strengths of our study include the large populationbased sample, the state-of-the-art assessment of BP and antihypertensive medication and the validation of cardiovascular events by an independent endpoint committee. We compared the population impact of Amerian, European and international hypertension guidelines for the first time, but there are also several limitations which remain to be considered. Our results are based on baseline data of the HNR study cohort, which is representative of the general population aged $45-75$ years living in German industrialised urban areas. Thus, generalisability to rural areas or other ethnicities remains to be shown. BP was only measured on one study visit, whereas definition and treatment of hypertension should ideally be based on multiple measurements of office BP or longterm ambulatory or home BP measurement. The HNR study is an observational study in which the exposure to guideline recommendation is not controlled by the investigator but by the treating physicians and the compliance of the patients, which can lead to the problem of bias and confounding prohibiting causal interpretation. The current gold standard method to identify causes of health outcomes is still the RCT. Our results call for additional RCTs to investigate whether applying stricter BP control recommendations leads to a reduction in cardiovascular events.

\section{Author affiliations}

${ }^{1}$ Department of Neurology, University Hospital Essen, Essen, Germany ${ }^{2}$ Institute for Medical Informatics, Biometry and Epidemiology, University Hospital Essen, University Duisburg-Essen, Essen, Germany

${ }^{3}$ Department of Epidemiology, Boston University School of Public Health, Boston, Massachusetts, USA

${ }^{4}$ West German Heart and Vascular Center Essen, Department of Cardiology and Vascular Medicine, University Hospital Essen, Essen, Germany

${ }^{5}$ Institute for Stroke and Dementia Research, University Hospital, Ludwig-

Maximilians-Universität LMU, Munich, Germany

${ }^{6}$ Munich Cluster of Systems Neurology (SyNergy), Munich, Germany

${ }^{7}$ Department of Angiology, Helios-Klinikum Krefeld, Krefeld, Germany

${ }^{8}$ Environmental Epidemiology Group, Institute of Occupational, Social and Environmental Medicine, Medical Faculty, Heinrich Heine University Düsseldorf, Düsseldorf, Germany

Correction notice This article has been corrected since it was first published. Middle initials has been added for the authors Amir A Mahabadi and Dirk M Hermann.

Acknowledgements The authors are indebted to all study participants, the dedicated study centre personnel and the investigative group.

Contributors JG analysed the data and wrote the manuscript. RK, NL and DH made substantial contributions to data interpretation, revised the manuscript critically for intellectual content and had final approval of the version to be published. AM, CW, MD, KK, SM and BH made substantial contributions to data acquisition, revised the manuscript critically for intellectual content, and had final approval of the version to be published. AS, K-HJ and RE made substantial contributions to study conception, revised the manuscript critically for intellectual content, and had final approval of the version to be published. JG is the guarantor.

Funding The authors thank the Heinz Nixdorf Foundation for their generous support. This study is also supported by the German Ministry of Education and Science (BMBF) and German Research Council (ER-155/6-2).

Disclaimer The funders of the study had no role in study design, data collection, data analysis, data interpretation, or writing of the report.

Competing interests None declared.

Patient consent for publication Not required.

Ethics approval The study complies with the Declaration of Helsinki and was approved by the ethical committee of the University of Duisburg-Essen, Germany (ID 99-69-1200).

Provenance and peer review Not commissioned; externally peer reviewed.

Data availability statement Data are available upon reasonable request. The corresponding author has full access to all data in the study and final responsibility for the submission of the article for publication. Due to data security reasons (ie, data contain potentially participant identifying information), the HNR Study does not allow sharing data as a public use file. Data requests can also be addressed to: recall@uk-essen.de.

Supplemental material This content has been supplied by the author(s). It has not been vetted by BMJ Publishing Group Limited (BMJ) and may not have been peer-reviewed. Any opinions or recommendations discussed are solely those of the author(s) and are not endorsed by BMJ. BMJ disclaims all liability and responsibility arising from any reliance placed on the content. Where the content includes any translated material, BMJ does not warrant the accuracy and reliability of the translations (including but not limited to local regulations, clinical guidelines, terminology, drug names and drug dosages), and is not responsible for any error and/or omissions arising from translation and adaptation or otherwise.

Open access This is an open access article distributed in accordance with the Creative Commons Attribution Non Commercial (CC BY-NC 4.0) license, which permits others to distribute, remix, adapt, build upon this work non-commercially, and license their derivative works on different terms, provided the original work is 
properly cited, appropriate credit is given, any changes made indicated, and the use is non-commercial. See: http://creativecommons.org/licenses/by-nc/4.0/.

\section{ORCID iD}

Janine Gronewold http://orcid.org/0000-0002-1470-424X

\section{REFERENCES}

1 Report of the joint National Committee on detection, evaluation, and treatment of high blood pressure. A cooperative study. JAMA 1977;237:255-61.

2 Chobanian AV, Bakris GL, Black HR, et al. The seventh report of the joint National Committee on prevention, detection, evaluation, and treatment of high blood pressure: the jnc 7 report. JAMA 2003;289:2560-72.

3 Whelton PK, Carey RM, Aronow WS, et al. 2017 acc/aha/aapa/abc/ acpm/ags/apha/ash/aspc/nma/pcna guideline for the prevention, detection, evaluation, and management of high blood pressure in adults: a report of the American College of cardiology/american heart association Task force on clinical practice guidelines. Circulation 2018;138:e484-594.

4 Muntner P, Carey RM, Gidding S, et al. Potential US population impact of the 2017 acc/aha high blood pressure guideline. Circulation 2018;137:109-18.

5 Mancia G, Fagard R, Narkiewicz K. esh/esc guidelines for the management of arterial hypertension: the task force for the management of arterial hypertension of the European Society of hypertension (esh) and of the European Society of cardiology (ESC). $J$ Hypertens 2013;2013:1281-357.

6 Williams B, Mancia G, Spiering W. esc/esh guidelines for the management of arterial hypertension: the task force for the management of arterial hypertension of the European Society of cardiology and the European Society of hypertension: the task force for the management of arterial hypertension of the European Society of cardiology and the European Society of hypertension. $J$ Hypertens 2018;2018:1953-2041.

7 Whitworth JA. World health organization (who)/international society of hypertension (ish) statement on management of hypertension. $J$ Hypertens 2003;2003:1983-92.

8 Unger T, Borghi C, Charchar F. International Society of hypertension global hypertension practice guidelines. Hypertension 2020;2020:1334-57.

9 Hyman DJ, Pavlik VN. Self-Reported hypertension treatment practices among primary care physicians: blood pressure thresholds, drug choices, and the role of guidelines and evidence-based medicine. Arch Intern Med 2000;160:2281-6.

10 Gronewold J, Kropp R, Lehmann N, et al. Cardiovascular risk and atherosclerosis progression in hypertensive persons treated to blood pressure targets. Hypertension 2019;74:1436-47.

11 Schmermund A, Möhlenkamp S, Stang A, et al. Assessment of clinically silent atherosclerotic disease and established and novel risk factors for predicting myocardial infarction and cardiac death in healthy middle-aged subjects: rationale and design of the heinz Nixdorf recall study. risk factors, evaluation of coronary calcium and lifestyle. Am Heart J 2002;144:212-8.

12 WHOCC. Whocc-structure and principles, 2017.

13 Holle R, Happich M, Löwel H, et al. KORA--a research platform for population based health research. Gesundheitswesen 2005;67 Suppl $1: 19-25$.
14 Lehmann N, Erbel R, Mahabadi AA, et al. Value of progression of coronary artery calcification for risk prediction of coronary and cardiovascular events: result of the hnr study (heinz nixdorf recall). Circulation 2018;137:665-79.

$15 \mathrm{lbm}$ corp. Ibm spss statistics for windows, version 22.0. Armonk, ny: lbm corp, 2013.

16 Goff DC, Lloyd-Jones DM, Bennett G, et al. 2013 ACC/AHA guideline on the assessment of cardiovascular risk: a report of the American College of Cardiology/American heart association Task force on practice guidelines. Circulation 2014;129:S49-73.

17 Vaucher J, Marques-Vidal P, Waeber G, et al. Population impact of the 2017 ACC/AHA guidelines compared with the $2013 \mathrm{ESH} /$ ESC guidelines for hypertension management. Eur J Prev Cardiol 2018;25:1111-3.

18 Vasan RS, Larson MG, Leip EP, et al. Impact of high-normal blood pressure on the risk of cardiovascular disease. $N$ Engl J Med 2001;345:1291-7.

19 , Cushman WC, Evans GW, et al, ACCORD Study Group. Effects of intensive blood-pressure control in type 2 diabetes mellitus. $N$ Engl $J$ Med 2010;362:1575-85.

20 Diao D, Wright JM, Cundiff DK, et al. Pharmacotherapy for mild hypertension. Cochrane Database Syst Rev 2012:Cd006742.

21 ESC. Governing policies and procedures for the writing of ESC clinical practice guidelines. Available: https://www.escardio.org/ static-file/Escardio/Guidelines/About/Recommendations-GuidelinesProduction.pdf

22 Foundation ACoC, Association AH. Methodology manual and policies from the accf/aha Task force on practice guidelines, 2010. Available: http://my.americanheart.org/idc/groups/ahamah-public/@ wcm/@sop/documents/downloadable/ucm_319826.pdf

23 Ettehad D, Emdin CA, Kiran A, et al. Blood pressure lowering for prevention of cardiovascular disease and death: a systematic review and meta-analysis. Lancet 2016;387:957-67.

24 Lonn EM, Bosch J, López-Jaramillo P, et al. Blood-Pressure lowering in intermediate-risk persons without cardiovascular disease. $N$ Engl J Med 2016;374:2009-20.

25 Sundström J, Arima H, Jackson R, et al. Effects of blood pressure reduction in mild hypertension: a systematic review and metaanalysis. Ann Intern Med 2015;162:184-91.

26 Thomopoulos C, Parati G, Zanchetti A. Effects of blood pressure lowering on outcome incidence in hypertension. 1. overview, metaanalyses, and meta-regression analyses of randomized trials. $J$ Hypertens 2014;32:2285-95.

27 Ambrosius WT, Sink KM, Foy CG, et al. The design and rationale of a multicenter clinical trial comparing two strategies for control of systolic blood pressure: the systolic blood pressure intervention trial (sprint). Clin Trials 2014;11:532-46.

28 , Wright JT, Williamson JD, et al, SPRINT Research Group. A randomized trial of intensive versus standard blood-pressure control. N Engl J Med 2015;373:2103-16.

29 Williamson JD, Supiano MA, Applegate WB, et al. Intensive vs standard blood pressure control and cardiovascular disease outcomes in adults aged $\geq 75$ years: a randomized clinical trial. JAMA 2016;315:2673-82.

30 Yusuf S, Lonn E, Pais P, et al. Blood-Pressure and cholesterol lowering in persons without cardiovascular disease. $N$ Engl J Med 2016;374:2032-43.

31 Yusuf S, Bosch J, Dagenais G, et al. Cholesterol lowering in intermediate-risk persons without cardiovascular disease. $N$ Engl J Med 2016;374:2021-31. 